Published in final edited form as:

Curr Opin Nephrol Hypertens. 2010 September ; 19(5): 425-431. doi:10.1097/MNH.0b013e32833bec06.

\title{
Sodium-glucose transport: role in diabetes mellitus and potential clinical implications
}

\author{
Volker Vallon ${ }^{\mathrm{a}, \mathrm{b},{ }^{*}}$ and Kumar Sharma ${ }^{\mathrm{a}, \mathrm{c},{ }^{,}}$ \\ aDivision of Nephrology and Hypertension, Department of Medicine, University of California San \\ Diego/Veterans Affairs San Diego Healthcare System, San Diego \\ bDepartment of Pharmacology, University of California San Diego, California, USA \\ ${ }^{\circ}$ Center for Renal Translational Medicine, University of California San Diego/VA San Diego \\ Healthcare System, San Diego, California, USA
}

\begin{abstract}
Purpose of review-Current options for glycemic control is less than optimal in terms of efficacy and to reduce complications in the diabetic population. Selective inhibition of SGLT2 in the proximal tubule increases urinary glucose excretion thereby reducing plasma glucose levels, which may present a novel therapeutic approach.
\end{abstract}

Recent findings-SGLT2 inhibitors enhance glucose excretion and improve glycemic control in patients with type 2 diabetes in the absence of clinically relevant hypoglycemia or sustained changes in volume status or glomerular filtration rate. This is associated with lowering of body weight and may reduce systolic blood pressure. The increased glucosuria appears to increase the risk of genital infections but may not increase the risk of urinary tract infections.

Summary-The ability of SGLT2 inhibitors to reduce plasma glucose without inducing increased insulin secretion, clinically relevant hypoglycemia, or weight gain constitutes a major advance. The ability of increased glucose excretion provides a powerful means to treat caloric excess conditions. Important questions remain to be resolved and more clinical research is needed on the long-term effects of SGLT2 inhibition. Potential extrarenal effects need to be explored in order to determine the safety of these compounds. It also remains to be determined whether these drugs lower the toxicity of glucose directly on renal cells, independent of hyperglycemia, which may slow or prevent the progressive nature of diabetic nephropathy.

\section{Keywords}

albuminuria; diabetic kidney disease; glucose transport; glycemic control

Correspondence to Kumar Sharma, MD, Director, Center for Renal Translational Medicine, UCSD/VA San Diego Healthcare System, La Jolla, CA 92093-0711, USA, Tel: +1 858822 0860; fax: +1 858822 7483; kusharma@ ucsd.edu.

*Volker Vallon and Kumar Sharma contributed equally to the writing of the article. 


\section{Introduction}

Diabetes-associated chronic renal insufficiency is the primary cause of end-stage renal disease (ESRD), yet the pathophysiology is still incompletely understood. Good control of blood glucose levels is important in diabetic patients but difficult to achieve without the potential for hypoglycemia and weight gain. Moreover, existing therapies may not always reduce cardiovascular complications, indicating the need for new therapies with novel mechanisms of action. This review discusses the rationale for the current development of inhibitors of SGLT2 as new antidiabetic drugs and presents newly available clinical data.

\section{Glucose transport in the kidney}

The kidney reabsorbs large amounts of glucose, and by extension has a major part in maintaining the overall metabolic balance of the body. In healthy humans, about $180 \mathrm{~g} / \mathrm{day}$ of glucose are filtered through the renal glomerulus, more than $99 \%$ of which is reabsorbed along the tubular system. Transport of glucose across cell membranes is accomplished by two gene families: the facilitative glucose transporters - the GLUTs - and by an active sodium-dependent transport process mediated by the $\mathrm{Na}^{+}$-glucose cotransporters (SGLT). The latter process allows glucose to be accumulated in cells against the concentration gradient.

The SGLTs are a large family of membrane proteins involved in the transport of glucose, amino acids, vitamins, osmolytes, and some ions [1]. At least two sodium-coupled glucose transporters, SGLT1 and SGLT2, play an important role in the apical membrane of proximal tubular cells in the kidney. The low-capacity, high-affinity SGLT1 is mainly present in the gastrointestinal tract with a minor role in the kidney, whereas the high-capacity, low-affinity SGLT2 transporter is found primarily in the kidney, in which it is responsible for the majority of glucose reabsorption [1-4].

\section{SGLT2 and glucose reabsorption in the proximal renal tubule}

The high-capacity, low-affinity SGLT2 is found primarily in the apical membrane of the S1 segment of the proximal tubule, whereas the low-capacity, high-affinity SGLT1 is located more distally in the $\mathrm{S} 3$ segment of the proximal tubule. Two basolateral membrane glucose transporters facilitate transcellular glucose transport - the low-affinity GLUT2, which works in concert with SGLT2 in the S1 segment of the renal tubule, and the high-affinity GLUT1, which works with SGLT1 in the S3 segment $[5,6]$.

The phenotype of humans carrying gene mutations in the genes for SGLT1 (SLC5A1) and SGLT2 (SLC5A2) provided much of the evidence for the quantitative contribution of these proteins to renal glucose reabsorption. Gene mutation of SGLT1 has serious consequences for afflicted individuals, who experience glucose-galactose malabsorption, frequent watery diarrhea, and dehydration [7]. However, these individuals have little or no glucosuria. In contrast, those who have SGLT2 gene mutations have persistent renal glucosuria, with glucose excretion of up to $160 \mathrm{~g} /$ day in severe cases. A total of 44 different mutations have been described for the SCL5A2 gene, including missense and nonsense mutations, small deletions (in-frame and frame shift), and splicing mutations, scattered throughout the gene 
[8]. Although no other complications are known to be consistently associated with mutations of this gene, it is a rare genetic disorder and not well studied [9-12]. New free-flow micropuncture studies in mice lacking SGLT2 confirmed a lack of glucose reabsorption in the early proximal tubule and showed that fractional renal reabsorption of glucose is reduced compared with wild-type mice ( $36 \pm 8$ vs. $99.7 \pm 0.1 \%$ ) and varied inversely with the amount of filtered glucose in the knockout mice (between 60 and 10\%) [13]. Thus, SGLT2 plays a major role for renal glucose reabsorption.

\section{SGLT2 expression and diabetes mellitus}

SGLT2 reabsorbs almost all glucose that occurs in glomerular filtrate; however, there is a transport maximum $\left(T_{\mathrm{m}}\right)$ for glucose that depends on the level of protein expression. The issue of gene expression and the possibility of SGLT2 adaptation to hyperglycemia is an area rich for investigation, showing reduced [14], unchanged [15] or increased expression [16] in diabetic rodent models. In-vitro studies indicated that high glucose-induced oxidative stress inhibits $\mathrm{Na}^{+}$-glucose cotransporter activity in renal proximal tubule cells [17]. New studies in streptozotocin-diabetic rats suggested that the increase in renal SGLT2 mRNA and protein expression in diabetes is mediated by angiotensin II AT1 receptors [ $\left.18^{\circ}\right]$. Primary cultures of human exfoliated proximal tubular epithelial cells from fresh urine of patients with type 2 diabetes indicated an increased renal glucose uptake associated with increased expression of SGLT2 and GLUT2 compared with healthy control individuals [19]. Up-regulation of SGLT2 expression in diabetes may involve the transcription factor, hepatocyte nuclear factor (HNF)-1a [20]. New studies provided evidence that the transcriptional activity of both HNF-1 $\alpha$ and HNF- $3 \beta$ may contribute to the renal overexpression of GLUT2 mRNA in the kidney of diabetic rats $\left[21^{\circ}\right]$.

\section{Diabetic nephropathy and glucose transport}

Diabetes-associated chronic renal insufficiency is the primary cause of ESRD [22]. A number of pathways have been implicated in the development of diabetic nephropathy, including comorbid hypertension and the involvement of the renin angiotensin aldosterone system (RAAS) [22,23]. Other possible pathways include aldose-reductase-polyol, di-acyl glycerol-protein kinase C, advanced glycated end products (AGE), and the hexosamine pathway $[24,25]$. Many of the proximal pathways culminate in simulation of transforming growth factor beta (TGFb) and CTGF production leading to matrix accumulation and progressive scarring [26,27]. Data on the role that glucose transport may play are beginning to emerge.

Glomerular hyperfiltration is a cardinal feature of early diabetes mellitus and may predispose to the eventual development of diabetic nephropathy [28,29]. According to the 'tubulo-centric principle', the underlying mechanism(s) of diabetic hyperfiltration include(s) a primary increase in proximal reabsorption, which reduces delivery of $\mathrm{NaCl}$ to the macula densa [30-32]. The macula densa senses this decline in salt delivery as an error signal and elicits an increase in glomerular filtration rate (GFR), which offsets a portion of the original error signal through negative tubulo-glomerular feedback. In accordance, glomerular hyperfiltration is blunted in early diabetes in mice that lack an intact tubulo-glomerular 
feedback response [33]. Enhanced tubular growth and glucose reabsorption have been implicated in proximal hyper-reabsorption and glomerular hyperfiltration in early diabetes [30,32]. In addition to impaired tubular function, diabetes may have direct effects on the afferent arteriole to impair the autoregulatory response. The impaired afferent arteriolar response to increased perfusion pressure is likely contributed by local production of TGF- $\beta$ [34]. The afferent arteriole becomes dilated, increasing glomerular blood flow and causing distension of the capillary loop early on in kidney disease. In turn, this places stress on mesangial cells, glomerular capillary cells, and podocytes. Stress may lead to podocyte dysfunction or dropout. Microalbuminuria follows and may progress to overt proteinuria. This progression is paralleled by the accumulation of mesangial matrix and basement membrane, glomerular hypertrophy, and increased podocyte dropout. Ultimately, the patient develops glomerular sclerosis, tubulointerstitial disease, tubular atrophy and microvascular disease $[35,36]$ leading to ESRD. The early glomerular changes have been noted to occur in diabetic $\mathrm{db} / \mathrm{db}$ mice as well [35]. The role of SGLTs in these changes is not known.

\section{Current therapies for hyperglycemia in type 2 diabetes and unmet needs}

Current noninsulin-based therapies for type 2 diabetes mellitus (T2DM) include the alphaglucosidase inhibitors, incretin mimetics, insulin secretagogues, metformin, sulfonylureas, thiazolidinediones, and more recently glucagon like peptide-1 agonist and dipeptidylpeptidase-IV inhibitors. Given the progressive nature of T2DM, most patients eventually require combinations of oral antidiabetic medications [37]. The United Kingdom Prospective Diabetes Study (UKPDS) found that after 3 years, only $50 \%$ of patients were adequately controlled on a single medication, and that after 9 years, only $25 \%$ were controlled on monotherapy [38]. The reasons for the difficulty in achieving glycemic goals are complex. Although the reluctance of medical professionals and patients to intensify treatment may contribute, it seems likely that the progression of the underlying disease process, together with dose-limiting side effects of current therapies, including gastrointestinal effects, fluid retention, weight gain, and hypoglycemia, contribute to the poor glycemic control that is common in T2DM. Furthermore, recent studies have found that further intensive glycemia control (i.e. hemoglobin A1C <6.5\%) with existing therapies do not reduce cardiovascular complications [39] and may increase mortality. Given that present therapies may be difficult to provide ideal glycemic control without the potential for hypoglycemia, weight gain and that existing therapies may not always reduce cardiovascular complications, there is a clear need for new therapies with novel mechanisms of action.

\section{Nonselective SGLT inhibition}

SGLT inhibition offers the potential to decrease the deleterious effects of hyperglycemia while reducing blood glucose levels by inhibiting renal glucose reabsorption. Impressive results have been achieved in diabetic animal with the nonselective SGLT inhibitor, phlorizin [40], its derivative T-1095 and its metabolite T-1095A [41,42], including beneficial effects on blood glucose levels and $\mathrm{HbA}_{1 \mathrm{c}}$ levels, partial improvement of glucose tolerance and insulin resistance, and suppression of albuminuria and the expansion of the glomerular mesangial area in diabetic rodents $[41,43]$. Due to their effect on SGLT1, however, 
nonselective inhibitors can have unacceptable gastrointestinal side effects and did not proceed to clinical development.

\section{Selective SGLT2 inhibition}

Selective SGLT2 inhibitors offer the potential to primarily increase renal excretion of glucose and create a negative energy balance without affecting intestinal function. As inhibition of intestinal absorption of foods has not been accepted by many patients, additional methods to create a negative energy balance may offer strong advantages over present approaches. A major potential advantage is that these compounds will not have direct effects to stimulate insulin release and thus cellular glucose uptake. In the short term increased intracellular glucose uptake will reduce hyperglycemia; however, potentially chronic and persistent increased intracellular glucose uptake could lead to cell dysfunction. Selective SGLT2 inhibition may also provide renoprotection through beneficial effects on glycemia, glomerular hemodynamics, and glucose toxicity of tubular cells. Various SGLT2 inhibitors are currently in development as well as a novel antisense approach to inhibit SGLT2.

\section{ISIS 388626}

As shown in preliminary studies, ISIS 388626, a 12-nucleotide SGLT2 antisense oligonucleotide, reduces the mRNA expression of SGLT2 in the proximal tubule of rodents, dogs and nonhuman primates by up to $75-80 \%$ and increases glucosuria in these species and improves glycemic control in Zucker diabetic fatty rats [44,45]. ISIS is currently evaluating this compound in a phase 1 study designed to assess the safety and activity of the drug in healthy volunteers.

\section{Dapagliflozin}

The compound has a 1200-fold selectivity for SGLT2 over SGLT1 as compared with phlorizin's 10-fold selectivity [46]. Dapagliflozin did not significantly inhibit facilitative glucose transport in human adipocytes [41,47]. In-vivo studies demonstrated that a single dose of dapagliflozin lowers blood glucose levels in streptozotocin-induced diabetic rats [46]. Moreover, dapagliflozin induced significant, dose-dependent glucosuria in normal and ZDF rats, enhanced glucose tolerance in normal rats, and decreased hyperglycemia in ZDF rats after single oral doses and 2-week treatment. Furthermore, the compound caused a significant increase in the glucose utilization rate, which was accompanied by a significant reduction in glucose production and enhanced liver insulin sensitivity without inducing hypoglycemia. No body weight changes compared with vehicle-treated rates were observed [46].

More recently, the safety, tolerability, pharmacokinetics, and pharmacodynamics of dapagliflozin were evaluated in single-ascending dose (SAD; $2.5-500 \mathrm{mg}$ ) and multipleascending dose (MAD; $2.5-100 \mathrm{mg}$ daily for 14 days) studies in healthy individuals [48 ${ }^{\circ}$. The compound induced a dose-dependent glucosuria. Cumulative daily amounts of glucose excreted ranged from 18 to $62 \mathrm{~g}$ and were comparable between 1 and 14 days of treatment, with no apparent changes in glycemic parameters. Once-daily doses of approximately 20-50 
mg provided close-to-maximal SGLT2 inhibition for at least $24 \mathrm{~h}\left[48^{\circ}, 49\right]$. A subsequent multiple-dose study evaluated the safety and efficacy of dapagliflozin in type 2 diabetic patients, which were randomly assigned to one of five dapagliflozin doses $(2-50 \mathrm{mg})$, metformin extended release, or placebo for 12 weeks [50*0. Dapagliflozin induced significant glucosuria (52-85 g urinary glucose/day) and demonstrated glycemic improvements and greater weight loss vs. placebo $\left(\Delta \mathrm{HbA}_{1 \mathrm{c}}-0.55\right.$ to -0.90 vs. $-0.18 \%$; $\Delta$ fasting plasma glucose -16 to -31 vs. $-6 \mathrm{mg} / \mathrm{dl} ;-2.5$ to -3.4 vs. $-1.2 \mathrm{~kg}$ ). Dapagliflozin did not induce clinically significant changes in estimated GFR or volume status and modestly lowered systolic blood pressure and serum uric acid levels. Rates of genital infections were higher with dapagliflozin (especially the higher doses) vs. placebo, whereas bacterial urinary tract infections were not different [50*0. A randomized, double-blind, three-arm, parallel-group, placebo-controlled, 26-center trial was performed to determined whether dapagliflozin lowers hyperglycemia in patients with type 2 diabetes that is poorly controlled with high insulin doses and oral antidiabetic agents (OADs) [51*0. Patients were randomly assigned to placebo, $10 \mathrm{mg}$ dapagliflozin, or $20 \mathrm{mg}$ dapagliflozin, and OADs and $50 \%$ of their daily insulin dose. Under these conditions, 12 weeks of dapagliflozin decreased $\mathrm{HbA1C}$, produced better fasting and postprandial plasma glucose levels, and lowered body weight more than placebo. Overall, adverse events were balanced across all groups, although more genital infections occurred in the 20-mg dapagliflozin group than in the placebo group [51*0]. Currently, multiple phase III clinical trials with dapagliflozin (mostly as add-on therapies) are being performed.

\section{Sergliflozin}

The active entity of sergliflozin etabonate is a highly potent and selective inhibitor of SGLT2. In rats, mice and dogs, oral administration of sergliflozin etabonate results in increased urinary glucose excretion in a dose-dependent manner. It also significantly lowers plasma glucose in a dose-dependent manner when administered to streptozotocin-induced diabetic rats, Zucker fatty diabetic rats or KK-A(y) mice, which have symptoms of obesity and hyperinsulinemia, without stimulating insulin secretion or inducing hypoglycemia [5254].

More recently, the pharmacokinetics and pharmacodynamics of sergliflozin were evaluated following single oral dose administration of sergliflozin etabonate $(5-500 \mathrm{mg}$ ) in healthy volunteers and patients with $\mathrm{T} 2 \mathrm{DM}\left[55^{\circ}\right]$. In both populations, sergliflozin etabonate produced a dose-related glucosuria under fasting conditions and following glucose loading but did not appreciably affect urinary electrolyte excretion or fluid balance. Single doses of sergliflozin etabonate 5-500 mg were well tolerated, and there were no clinically significant adverse laboratory findings $\left[5^{\circ}\right]$. Studies with administration of sergliflozin etabonate (500 or $1000 \mathrm{mg}$ ) or placebo three times daily (t.i.d.) for 14 days in healthy overweight or obese human volunteers revealed a mean half-life of the active entity of approximately $2 \mathrm{~h}\left[56^{\circ}\right.$ ]. Sergliflozin etabonate induced a dose-related glucosuria ( 21 and $51 \mathrm{~g} /$ day) associated with unchanged plasma glucose, insulin, and electrolyte levels, whereas body weight was reduced [mean changes from baseline of $-1.55(500 \mathrm{mg})$ and $-1.74 \mathrm{~kg}(1000 \mathrm{mg}) \mathrm{vs} .-0.09 \mathrm{~kg}$ with placebo, respectively]. Sergliflozin etabonate did not alter creatinine clearance and was generally well tolerated. Excretion in the urine of (micro)albumin, and of the tubular injury 
markers, $\mathrm{N}$-acetyl-beta-D-glucosaminidase and $\beta 2$-microglobulin, were increased in several individuals receiving sergliflozin but the clinical relevance of these effects is uncertain $\left[56^{\circ}\right]$. The clinical development of sergliflozin etabonate had been discontinued in favor of remogliflozin.

Remogliflozin etabonate is a prodrug that is metabolized to its active form, the selective SGLT2 inhibitor, remogliflozin, in the body [57]. Orally administered remogliflozin etabonate increased urinary glucose excretion and showed antihyperglycemic effects in both streptozotocin-induced diabetic rats in oral glucose tolerance and in $\mathrm{db} / \mathrm{db}$ mice. In high-fat diet-fed Goto-Kakizaki rats, remogliflozin etabonate improved hyperglycemia, hyperinsulinemia, hypertriglyceridemia, and insulin resistance [57]. Preliminary studies in patients with type 2 diabetes supported the safety of its administration with metformin with minimal risk of hypoglycemia [58]. Further preliminary studies in these patients revealed a plasma half-life of $120 \mathrm{~min}$ and dose-dependent increases in urinary glucose excretion [59]. The clinical development of remogliflozin etabonate, however, has been discontinued in 2009 as a result of extenuating circumstances, including the development status of SGLT2 inhibitors by competitors.

Other SGLT2 inhibitors in development include AVE2268 [60], BI10773 [61], BI44847 [62], canagliflozin [62], LX4211 [62], ASP1941 [62] and TS-071 [63], but no clinical data are available or published yet.

\section{Implications of current findings}

On the basis of preclinical and the first clinical studies, SGLT2 inhibition shows promise as a potential therapy for improving glycemic control and possibly offering renoprotection in T2DM. The ability of a class of agents to reduce plasma glucose without inducing increased insulin secretion, clinically relevant hypoglycemia, or weight gain constitutes a major advance.

However, important questions remain to be resolved and more clinical research is needed on the long-term effects of SGLT2 inhibition on kidney function, blood pressure and bone health. The available studies find no increase in bacterial urinary tract infection in response to SGLT2 inhibition. The incidence of genital fungal infections, however, may be increased, which requires close observation. These drugs are lipid-soluble, and therefore will cross the blood brain barrier (BBB). The SGLTs are found within the hypothalamus in which they sense and respond to changes in glucose concentrations [64]. Real-time PCR suggested SGLT2 RNA expression in many human tissues [65] but, due to the lack of good antibodies, the implication for extrarenal protein expression of SGLT2 is unclear. It is critical that potential extra-renal effects are explored in order to determine the safety of these compounds.

Potential benefits of renoprotection must be investigated thoroughly as well. Do these drugs affect kidney growth and hyperfiltration in early diabetes? Does SGLT2 inhibition have direct effects on cells to inhibit matrix production and cell dysfunction? Studies are needed to determine if there are beneficial effects on cytokines such as TGF $\beta 1$ and CTGF to 
determine whether these compounds can slow or prevent the progressive nature of diabetic nephropathy. It will be challenging to prove that any benefits are over and above the reduction of systemic hyperglycemia. Ultimately, their effects in patients with early and established kidney disease must be explored. A preferential reduction in albuminuria and markers of tubular damage with SGLT2 inhibitors will be of major interest. Those questions notwithstanding, SGLT2 inhibition appears to offer substantial hope for a unique and effective diabetes therapy, with an acceptable adverse effect profile, and potential beneficial effects on the kidneys.

\section{Conclusion}

SGLT2 mediates glucose reabsorption in the early proximal tubule and the majority of the overall glucose reabsorption by the kidney. The available clinical studies show the ability of SGLT2 inhibitors to enhance the urinary excretion of glucose in patients with T2DM, thereby reducing plasma glucose levels without inducing increased insulin secretion, clinically relevant hypoglycemia, or weight gain. Benefits to diabetic kidney disease will likely be due to overall improved glycemic control without weight gain, and potentially direct benefit by inhibiting glucose uptake in renal cells.

\section{Acknowledgments}

Studies by the authors were supported by the National Institutes of Health (DK56248, DK28602, P30DK079337 to V.V.), (R01 DK 053867 and U01 DK 060995 to K.S.), the Department of Veterans Affairs (V.V. and K.S.), and by Bristol-Myers Squibb and Astra-Zeneca (V.V.).

\section{References and recommended reading}

Papers of particular interest, published within the annual period of review, have been highlighted as:

- of special interest

$\bullet$ of outstanding interest

Additional references related to this topic can also be found in the Current World Literature section in this issue (pp. 000-000).

1. Wright EM, Turk E. The sodium/glucose cotransport family SLC5. Pflugers Arch. 2004; 447:510518. [PubMed: 12748858]

2. You G, Lee WS, Barros EJ, et al. Molecular characteristics of $\mathrm{Na}(+)$-coupled glucose transporters in adult and embryonic rat kidney. J Biol Chem. 1995; 270:29365-29371. [PubMed: 7493971]

3. Wright EM. Renal Na(+)-glucose cotransporters. Am J Physiol Renal Physiol. 2001; 280:F10-F18. [PubMed: 11133510]

4. Kanai Y, Lee WS, You G, et al. The human kidney low affinity Na+/glucose cotransporter SGLT2. Delineation of the major renal reabsorptive mechanism for D-glucose J Clin Invest. 1994; 93:397404. [PubMed: 8282810]

5. Wood IS, Trayhurn P. Glucose transporters (GLUT and SGLT): expanded families of sugar transport proteins. Br J Nutr. 2003; 89:3-9. [PubMed: 12568659]

6. Chin E, Zhou J, Bondy C. Anatomical and developmental patterns of facilitative glucose transporter gene expression in the rat kidney. J Clin Invest. 1993; 91:1810-1815. [PubMed: 8473519] 
7. Wright, EM., Martin, MG., Turk, E. Familial glucose-galactose malabsorption and hereditary renal glycosuria. In: Scriver, CR.Beaudet, AL.Sly, WS., Valle, D., editors. Metabolic basis of inherited disease. 8th. New York: McGraw-Hill; 2001. p. 4891-4980.

8. Santer R, Calado J. Familial renal glucosuria and SGLT2: from a Mendelian trait to a therapeutic target. Clin J Am Soc Nephrol. 2010; 5:133-141. [PubMed: 19965550]

9. van den Heuvel LP, Assink K, Willemsen M, Monnens L. Autosomal recessive renal glucosuria attributable to a mutation in the sodium glucose cotransporter (SGLT2). Hum Genet. 2002; 111:544-547. [PubMed: 12436245]

10. Francis J, Zhang J, Farhi A, et al. A novel SGLT2 mutation in a patient with autosomal recessive renal glucosuria. Nephrol Dial Transplant. 2004; 19:2893-2895. [PubMed: 15496564]

11. Magen D, Sprecher E, Zelikovic I, Skorecki K. A novel missense mutation in SLC5A2 encoding SGLT2 underlies autosomal-recessive renal glucosuria and aminoaciduria. Kidney Int. 2005; 67:34-41. [PubMed: 15610225]

12. Scholl-Burgi S, Santer R, Ehrich JH. Long-term outcome of renal glucosuria type 0: the original patient and his natural history. Nephrol Dial Transplant. 2004; 19:2394-2396. [PubMed: 15299100]

13. Vallon V, Platt KA, Cunard R, et al. Lack of early proximal tubular glucose reabsorption in transgenic Sglt2 null mice. J Am Soc Nephrol. 2010 in press.

14. Albertoni Borghese MF, Majowicz MP, Ortiz MC, et al. Expression and activity of SGLT2 in diabetes induced by streptozotocin: relationship with the lipid environment. Nephron Physiol. 2009; 112:45-52.

15. Adachi T, Yasuda K, Okamoto Y, et al. T-1095, a renal Na+-glucose transporter inhibitor, improves hyperglycemia in streptozotocin-induced diabetic rats. Metabolism. 2000; 49:990-995. [PubMed: 10954015]

16. Vestri S, Okamoto MM, De Freitas HS, et al. Changes in sodium or glucose filtration rate modulate expression of glucose transporters in renal proximal tubular cells of rat. J Membr Biol. 2001; 182:105-112. [PubMed: 11447502]

17. Han HJ, Lee YJ, Park SH, et al. High glucose-induced oxidative stress inhibits Na+/glucose cotransporter activity in renal proximal tubule cells. Am J Physiol Renal Physiol. 2005; 288:F988F996. [PubMed: 15598843]

18•. Osorio H, Bautista R, Rios A, et al. Effect of treatment with losartan on salt sensitivity and SGLT2 expression in hypertensive diabetic rats. Diabetes Res Clin Pract. 2009; 86:e46-e49. This study indicates a role for angiotensin II AT1 receptors in the up-regulation of SGLT2 in the diabetic kidney. [PubMed: 19800706]

19. Rahmoune H, Thompson PW, Ward JM, et al. Glucose transporters in human renal proximal tubular cells isolated from the urine of patients with noninsulin-dependent diabetes. Diabetes. 2005; 54:3427-3434. [PubMed: 16306358]

20. Freitas HS, Anhe GF, Melo KF, et al. $\mathrm{Na}(+)$-glucose transporter-2 messenger ribonucleic acid expression in kidney of diabetic rats correlates with glycemic levels: involvement of hepatocyte nuclear factor-1alpha expression and activity. Endocrinology. 2008; 149:717-724. [PubMed: 17962340]

21 . Freitas HS, Schaan BD, David-Silva A, et al. SLC2A2 gene expression in kidney of diabetic rats is regulated by HNF-1alpha and HNF-3beta. Mol Cell Endocrinol. 2009; 305:63-70. This study indicates a potential role of hepatocyte nuclear factor-1a in the regulation of GLUT2, which is co-expressed with SGLT2 in the early proximal tubule. [PubMed: 19433262]

22. Prasad P, Tiwari AK, Kumar KM, et al. Chronic renal insufficiency among Asian Indians with type 2 diabetes: I. Role of RAAS gene polymorphisms. BMC Med Genet. 2006; 7:42. [PubMed: 16672053]

23. Vieitez P, Gomez O, Uceda ER, et al. Systemic and local effects of angiotensin II blockade in experimental diabetic nephropathy. J Renin Angiotensin Aldosterone Syst. 2008; 9:96-102. [PubMed: 18584585]

24. Tiwari AK, Prasad P, BK T, et al. Oxidative stress pathway genes and chronic renal insufficiency in Asian Indians with type 2 diabetes. J Diabetes Complications. 2009; 23:102-111. [PubMed: 18413200] 
25. Prasad P, Tiwari AK, Kumar KM, et al. Association analysis of ADPRT1, AKR1B1, RAGE, GFPT2 and PAI-1 gene polymorphisms with chronic renal insufficiency among Asian Indians with type-2 diabetes. BMC Med Genet. 2010; 11:52. [PubMed: 20353610]

26. Zhu Y, Usui HK, Sharma K. Regulation of transforming growth factor beta in diabetic nephropathy: implications for treatment. Semin Nephrol. 2007; 27:153-160. [PubMed: 17418684]

27. Prasad P, Tiwari AK, Kumar KM, et al. Association of TGFbeta1, TNFalpha, CCR2 and CCR5 gene polymorphisms in type- 2 diabetes and renal insufficiency among Asian Indians. BMC Med Genet. 2007; 8:20. [PubMed: 17428349]

28. Mogensen CE, Christensen CK. Predicting diabetic nephropathy in insulin-dependent patients. N Engl J Med. 1984; 311:89-93. [PubMed: 6738599]

29. Mogensen CE. Early glomerular hyperfiltration in insulin-dependent diabetics and late nephropathy. Scand J Clin Lab Invest. 1986; 46:201-206. [PubMed: 3715374]

30. Vallon V, Richter K, Blantz RC, et al. Glomerular hyperfiltration in experimental diabetes mellitus: potential role of tubular reabsorption. J Am Soc Nephrol. 1999; 10:2569-2576. [PubMed: 10589696]

31. Vallon V, Blantz RC, Thomson S. Glomerular hyperfiltration and the salt paradox in early type 1 diabetes mellitus: a tubulo-centric view. J Am Soc Nephrol. 2003; 14:530-537. [PubMed: 12538755]

32. Thomson SC, Deng A, Bao D, et al. Ornithine decarboxylase, kidney size, and the tubular hypothesis of glomerular hyperfiltration in experimental diabetes. J Clin Invest. 2001; 107:217224. [PubMed: 11160138]

33. Vallon V, Schroth J, Satriano J, et al. Adenosine A(1) receptors determine glomerular hyperfiltration and the salt paradox in early streptozotocin diabetes mellitus. Nephron Physiol. 2009; 111:30-38.

34. Sharma K, Cook A, Smith M, et al. TGF-beta impairs renal autoregulation via generation of ROS. Am J Physiol Renal Physiol. 2005; 288:F1069-F1077. [PubMed: 15644487]

35. Sharma K, McCue P, Dunn SR. Diabetic kidney disease in the $\mathrm{db} / \mathrm{db}$ mouse. Am J Physiol Renal Physiol. 2003; 284:F1138-F1144. [PubMed: 12736165]

36. Lemley KV. Diabetes and chronic kidney disease: lessons from the Pima Indians. Pediatr Nephrol. 2008; 23:1933-1940. [PubMed: 18320236]

37. Inzucchi SE. Oral antihyperglycemic therapy for type 2 diabetes: scientific review. J Am Med Assoc. 2002; 287:360-372.

38. Turner RC, Cull CA, Frighi V, Holman RR. Glycemic control with diet, sulfonylurea, metformin, or insulin in patients with type 2 diabetes mellitus: progressive requirement for multiple therapies (UKPDS 49). UK Prospective Diabetes Study (UKPDS) Group. J Am Med Assoc. 1999; 281:2005-2012.

39. Gerstein HC, Miller ME, Byington RP, et al. Effects of intensive glucose lowering in type 2 diabetes. N Engl J Med. 2008; 358:2545-2559. [PubMed: 18539917]

40. Ehrenkranz JR, Lewis NG, Kahn CR, Roth J. Phlorizin: a review. Diabetes Metab Res Rev. 2005; 21:31-38. [PubMed: 15624123]

41. Ueta K, Ishihara T, Matsumoto Y, et al. Long-term treatment with the Na+-glucose cotransporter inhibitor T-1095 causes sustained improvement in hyperglycemia and prevents diabetic neuropathy in Goto-Kakizaki Rats. Life Sci. 2005; 76:2655-2668. [PubMed: 15792833]

42. Arakawa K, Ishihara T, Oku A, et al. Improved diabetic syndrome in C57BL/KsJ-db/db mice by oral administration of the $\mathrm{Na}(+)$-glucose cotransporter inhibitor T-1095. Br J Pharmacol. 2001; 132:578-586. [PubMed: 11159708]

43. Oku A, Ueta K, Arakawa K, et al. T-1095, an inhibitor of renal Na+-glucose cotransporters, may provide a novel approach to treating diabetes. Diabetes. 1999; 48:1794-1800. [PubMed: 10480610]

44. Wancewicz E, Bernd A, Meibohm B, et al. Long-term safety and efficacy of ISIS-388626, an optimized SGLT2 antisense inhibitor, in multiple diabetic and euglycemic species. Diabetes. 2008; 57(Suppl):A96. (Abstract).

45. Bhanot, S., Murray, S., Booten, S., et al. Proc of the 69th Scientific Session of the American Diabetes Association 2009. New Orleans, LA: ISIS 388626, an SGLT2 antisense drug, causes 
robust and sustained glucosuria in multiple species and is safe and well tolerated. (Abstract 328OR)

46. Meng W, Ellsworth BA, Nirschl AA, et al. Discovery of dapagliflozin: a potent, selective renal sodium-dependent glucose cotransporter 2 (SGLT2) inhibitor for the treatment of type 2 diabetes. J Med Chem. 2008; 51:1145-1149. [PubMed: 18260618]

47. Han S, Hagan DL, Taylor JR, et al. Dapagliflozin, a selective SGLT2 inhibitor, improves glucose homeostasis in normal and diabetic rats. Diabetes. 2008; 57:1723-1729. [PubMed: 18356408]

48• Komoroski B, Vachharajani N, Boulton D, et al. Dapagliflozin, a novel SGLT2 inhibitor, induces dose-dependent glucosuria in healthy subjects. Clin Pharmacol Ther. 2009; 85:520-526. This study shows the safety, tolerability, pharmacokinetics, and pharmacodynamics of dapagliflozin in healthy individuals. [PubMed: 19129748]

49. Obermeier M, Yao M, Khanna A, et al. In vitro characterization and pharmacokinetics of dapagliflozin (BMS-512148), a potent sodium-glucose cotransporter type II inhibitor, in animals and humans. Drug Metab Dispos. 2010; 38:405-414. [PubMed: 19996149]

$50 \bullet$. List JF, Woo V, Morales E, et al. Sodium-glucose cotransport inhibition with dapagliflozin in type 2 diabetes. Diabetes Care. 2009; 32:650-657. This study shows the safety and efficacy of dapagliflozin in type 2 diabetic patients. [PubMed: 19114612]

51•. Wilding JP, Norwood P, T'joen C, et al. A study of dapagliflozin in patients with type 2 diabetes receiving high doses of insulin plus insulin sensitizers: applicability of a novel insulinindependent treatment. Diabetes Care. 2009; 32:1656-1662. This study shows that dapagliflozin lowers hyperglycemia in patients with type 2 diabetes that is poorly controlled with high insulin doses and oral antidiabetic agents. [PubMed: 19528367]

52. Katsuno K, Fujimori Y, Takemura Y, et al. Sergliflozin, a novel selective inhibitor of low-affinity sodium glucose cotransporter (SGLT2), validates the critical role of SGLT2 in renal glucose reabsorption and modulates plasma glucose level. J Pharmacol Exp Ther. 2007; 320:323-330. [PubMed: 17050778]

53. Fujimori Y, Katsuno K, Ojima K, et al. Sergliflozin etabonate, a selective SGLT2 inhibitor, improves glycemic control in streptozotocin-induced diabetic rats and Zucker fatty rats. Eur J Pharmacol. 2009; 609:148-154. [PubMed: 19281809]

54. Katsuno K, Fujimori Y, Ishikawa-Takemura Y, Isaji M. Long-term treatment with sergliflozin etabonate improves disturbed glucose metabolism in KK-A(y) mice. Eur J Pharmacol. 2009; 618:98-104. [PubMed: 19615995]

55. Hussey EK, Clark RV, Amin DM, et al. Single-dose pharmacokinetics and pharmacodynamics of sergliflozin etabonate, a novel inhibitor of glucose reabsorption, in healthy volunteers and patients with type 2 diabetes mellitus. J Clin Pharmacol. 2010 [Epub ahead of print]. This study establishes the pharmacokinetics and pharmacodynamics of a single oral dose of sergliflozin etabonate in healthy volunteers and patients with type 2 diabetes mellitus.

56••. Hussey EK, Dobbins RL, Stoltz RR, et al. Multiple-dose pharmacokinetics and pharmacodynamics of sergliflozin etabonate, a novel inhibitor of glucose reabsorption, in healthy overweight and obese subjects: a randomized double-blind study. J Clin Pharmacol. 2010 [Epub ahead of print]. The study shows the efficacy of sergliflozin etabonate given for 14 days in healthy overweight or obese human volunteers.

57. Fujimori Y, Katsuno K, Nakashima I, et al. Remogliflozin etabonate, in a novel category of selective low-affinity sodium glucose cotransporter (SGLT2) inhibitors, exhibits antidiabetic efficacy in rodent models. J Pharmacol Exp Ther. 2008; 327:268-276. [PubMed: 18583547]

58. Hussey, EK., O'Connor-Semmes, RL., Tao, W., et al. Proc of the 69th Scientific Session of the American Diabetes Association 2009. New Orleans: Safety, pharmacokinetics and pharmacodynamics of remogliflozin etabonate (SGLT2 inhibitor) and metformin when coadministered in type 2 diabetes mellitus patients. (Abstract 509-P)

59. Kapur, A., Hussey, EK., Dobbins, RL., et al. Proc of the 69th Scientific Session of the American Diabetes Association 2009. New Orleans: First human dose escalation study with remogliflozin etabonate (RE) in healthy subjects and in subjects with type 2 diabetes mellitus. (Abstract 509-P)

60. Bickel M, Brummerhop H, Frick W, et al. Effects of AVE2268, a substituted glycopyranoside, on urinary glucose excretion and blood glucose in mice and rats. Arzneimittelforschung. 2008; 58:574-580. [PubMed: 19137908] 
61. Grempler, R., Thomas, L., Eckhardt, M., et al. Proc of the 69th Scientific Session of the American Diabetes Association 2009. New Orleans: In vitro properties and in vivo effect on urinary glucose excretion of BI 10773, a novel selective SGLT2 inhibitor. (Abstract 521-P)

62. US National Institutes of Health. ClinicalTrials.gov [online]. 2010. Available from URL. http:// www.clinicaltrials.gov

63. Kakinuma H, Oi T, Hashimoto-Tsuchiya Y, et al. (1S)-1,5-anhydro-1-[5-(4-ethoxybenzyl)-2methoxy-4-methylphenyl]-1-thio-D-glucitol (TS-071) is a potent, selective sodium-dependent glucose cotransporter 2 (SGLT2) inhibitor for type 2 diabetes treatment. J Med Chem. 2010; 53:3247-3261. [PubMed: 20302302]

64. O’Malley D, Reimann F, Simpson AK, Gribble FM. Sodium-coupled glucose cotransporters contribute to hypothalamic glucose sensing. Diabetes. 2006; 55:3381-3386. [PubMed: 17130483]

65. Zhou L, Cryan EV, D'Andrea MR, et al. Human cardiomyocytes express high level of Na+/glucose cotransporter 1 (SGLT1). J Cell Biochem. 2003; 90:339-346. [PubMed: 14505350] 